Neurosurg Focus 22 (4):E(Intro), 2007

\title{
Hydrocephalus in adults
}

\author{
Harold L. Rekate, M.D. \\ Pediatric Neurosciences, Barrow Neurological Institute, Phoenix; and University of Arizona School of \\ Medicine, Tucson, Arizona
}

$\mathrm{T}$ HE CURRENT ISSUE of Neurosurgical Focus is devoted to studies of the diagnosis and treatment of hydrocephalus and emphasizes the importance of those issues in adults. Little has been published about this group of patients except as relates to normal-pressure hydrocephalus (NPH), which has become a major focus of study. Its treatment has been greeted with enthusiasm as a result of improvements in technology.

An epidemiological study by Marmarou and associates was conducted in nursing homes in Vi rginia to identify individuals who might benefit from treatment of hydrocephalus, particularly NPH. Of 147 patients residing in nursing homes, 17 were selected as possibly having NPH and underwent screening according to the Medical College of Virginia protocol. Eleven patients underwent placement of a shunt, and seven, or $5 \%$ of the total nursing home population studied, improved significantly. The take-home message is that these patients had NPH for a number of years and would have enjoyed an improved quality of life had the disease been diagnosed and treated earlier.

The articles in this issue include a discussion of the utility of mathematical modeling in the study of the pathophysiology of hydrocephalus (Clarke and Meyer) and of the value of the pulsatility of intracranial pressure (ICP) waves to improve our understanding of this condition (Czosnyka and colleagues). The amplitude of the pulse wave confirms that the monitor is actually recording ICP. It also is a potential source of information that may help improve the selection of patients for treatment, especially when differentiating between brain atrophy and chronic compensated hydrocephalus, also known as "long-standing overt ventriculomegaly in adults." Chronic compensated hydrocephalus is almost certainly the substrate for the later development of NPH. ${ }^{1,2}$ Mounting evidence suggests that early management of this condition can improve outcomes and possibly avoid the need to institutionalize as many as $5 \%$ of elderly patients. ${ }^{3,4}$

When to begin treatment of patients who will eventually suffer NPH is a function of the level of safety that can be expected from treatment. Several articles in this issue deal with technological improvements in treatment, including the prevention of infection. Authors of a retrospective study (Sciubba et al.) showed that the use of anti- biotic-impregnated catheters decreased the rate of infection from 12 to $1.4 \%$. Differential pressure shunts lead to subatmospheric ICP unless the overdrainage associated with fixed pressure valves can be prevented. In one article Kurtom and Magram describe various strategies to prevent overdrainage and discuss the physical principles of these devices and their limitations in the treatment of hydrocephalus.

Treating hydrocephalus in adults is complicated by decisions that are much more rare in children. Should the surgeon intervene in the context of adult hydrocephalus without overt intracranial hypertension? If intervention is pursued and fails, are other treatment options still available? Geocadin and colleagues extended their previous study on the diagnosis of shunt failure in which they showed that patients who are never better or who decline later without signs of increased ICP should undergo shunt revision and are likely to improve thereafter. ${ }^{5}$ In the study presented here, they found that shunt failure can be diagnosed in this subtle group by chronic ICP monitoring and not by acute shunt tap or imaging studies.

In summary, there is much to learn about decision making in the treatment of hydrocephalus in adults. The articles presented here show the areas in which research and study will bear fruit.

\section{References}

1. Bradley WG, Safar FG, Hurtado C, Ord J, Alksne JF: Increased intracranial volume: a clue to the etiology of idiopathic normalpressure hydrocephalus? AJNR Am J Neuroradiol 25: 1479-1484, 2004

2. Bradley WG Jr, Bahl G, Alksne JF: Idiopathic normal pressure hydrocephalus may be a "two hit" disease: benign external hydrocephalus in infancy followed by deep white matter ischemia in late adulthood. J Magn Reson Imaging 24:747-755, 2006

3. Larsson A, Stephensen H, Wikkelso C: Adult patients with "asymptomatic" and "compensated" hydrocephalus benefit from surgery. Acta Neurol Scand 99:81-90, 1999

4. Tisell M, Tullberg M, Hellstrom P, Blomsterwall E, Wikkelso C: Neurological symptoms and signs in adult aqueductal stenosis. Acta Neurol Scand 107:311-317, 2003

5. Williams MA, Razumovsky AY, Hanley DF: Evaluation of shunt function in patients who are never better, or better than worse after shunt surgery for NPH. Acta Neurochir Suppl 71:368-370, 1998 